\title{
Phytomedicinal Flora and their Folk claim of Visakha Patnam District Agency, Andhra Pradesh, India.
}

\author{
S. B. Padal ${ }^{1}$ and K. Satya vathi ${ }^{2}$ \\ 1. Department of Botany, Mrs. A.V.N. College, Visakhapatnam District. Andhra Pradesh, India. \\ 2. Department of botany, Govt. Degree Dr. V. S. Krishna College, Visakhapatnam District. Andhra Pradesh, \\ India.
}

\begin{abstract}
The phytomedicinal plants study was carried out in adjoining tribal areas of Visakha patnam District, Andhra Pradesh during the month of November - December 2011. The information related to medicinal species which are used to cure common ailments and diseases were collected by the local people of study area. A total of 100 plants belonging to 53 families are listed in this paper.
\end{abstract}

Key Words: Agency area, Phytomedicinal flora, Traditional uses, Tribal people, Visakhapatnam.

\section{Introduction}

The use of plants as a relief for human suffering is as old as human especially, in India and China; people are using plants in organized healthcare for over 5000 years. Local communities mainly depend on traditional remedies, largely based on plants, for immediate access to relatively safe, cost effective, efficacious and culturally acceptable solutions to primary health care. In the oral traditions, local communities of all ecosystems right from trans-Himalayas down to costal plains have discovered the medicinal uses of thousands of plants found locally. India has one of the richest plant medicinal cultures in the world. This is a culture of tremendous contemporary relevance because, it can on one hand ensure health security to millions of people on the other hand it can provide new and safe herbal drugs to the entire world. World Health Organization (WHO) notes that out of 119 plants derived Pharmaceutical medicines, about 747 are used in modern medicine in way that correlated directly with their traditional uses as plant medicines by native cultures. C.S. Reddy et al. 2000, contains a note on medicinal uses of Hildegard populifolia and Pterocarpus santalinus: Red listed and endemic taxa in Andhra Pradesh. S.N. Jadhav et al. (2001) proceedings of the workshop on conservation Assessment and Management Planning (CAMP) for medicinal plants of Andhra Pradesh. C.S. Reddy et al. (2001) enumerated the threatened medicinal plants of Andhra Pradesh. R. Jeevan \& Raju. (2001) described certain potential crude drugs used by tribals of Nallamalais, Andhra Pradesh for Skin diseases. K.N. Reddy et al. (2002) reported the ethnobotany of some of the orchids of Andhra Pradesh while S.N. Jadhav \& K.N. Reddy (2002) presents a paper on In-Situ Conservation of Medicinal Plants in Andhra Pradesh. Banerjee (1977) \& Gupta et al. (1997) has reported the ethnobotany of Araku valley in Visakhapatnam district. T.A. Reddy (1980) note down some medicinal plants of Polavaram Agency, West Godavari district. Nisteswar \& Kumar $(1980,1983)$ reported the phytomedicine from Rampa and Addateegala Agency, East Godavari district. Rao \& Harasreeramulu (1985) described the selected medicinal plants of Srikakulam district. Sudhakar \& Rao (1985) enlisted the medicinal plants of East Godavari while Aruneekumar et al. (1990) enumerated the medicinal plants of Kakinada.

\section{Study Area}

Visakhapatnam district is one of the North Eastern Coastal district of Andhra Pradesh and it lies between $17^{\circ}-15^{1}$ and $18^{\circ}-32^{1}$ Northern latitude and $18^{\circ}-54^{1}$ and $83^{\circ}-30^{1}$ in Eastern longitudes. It is bounded on the North partly by the Orissa State and partly by Vizianagaram District, on the South by East Godavari District, on the West by Orissa State and on the East by Bay of Bengal. The district presents 2 distinct Geographic divisions. The strip of the land along the coast and the interior called the plains division and hilly area of the Eastern Ghats flanking it on the North and West called the Agency Division. The Agency Division consists of the hilly regions covered by the Eastern Ghats with an altitude of about 900 metres dotted by several peaks exceeding 1200 metres. Sankaram Forest block topping with 1615 metres embraces the mandals of paderu, G. Madugula, Hukumpeta Chintapalli, G.K. Veedhi, Koyyuru, Pedabayalu, Munchingiput, Dumbriguda, Arakuvalley and Ananthgiri. Visakha patnam district is rich in its forest resources. The agency areas with thick forests on hills, on hill slopes and in valleys. The total area under forest cover in this area is $104811.91 \mathrm{Ha}$. As against the total extent of 3,24,965 Ha. Of the division. The luxuriant forests in the Anantagiri, Araku, Minimuluru, G. Madugula, Chinta palli, and Gudem kothaveedi areas present a good sight to any tourist or naturalist. Based on Champion' and Seth (1968) classification, the forests in the agency area can be divided in to the following types.

1. Southern Tropical Semi-evergreen Forests: 
2. Southern Tropical Moist Deciduous Forests:

3. Savannahs or Hill Top Forest:

4. Southern Tropical Dry Deciduous forests:

The climate of the agency area is classified as Sub-tropical with high seasonal variation in rainfall and wide extremes of temperature. The average minimum temperature ranges from $3^{0}$ to $4^{0} \mathrm{C}$ in November/December while average maximum temperature ranges from $35^{\circ}$ to $40^{\circ} \mathrm{C}$ in May/June. Regarding rainfall and seasonal conditions usually the southwest monsoon starts from $3^{\text {rd }}$ week of April every year and northeast monsoon starts from October.

\section{Methodology}

The study was conducted during the month of November - December 2011 in tribal area of Visakha Patnam district. The information on local use and diseases cured was collected using structured questionnaire from the local people. The plant collections were identified with the help of Flora of Andhra Pradesh and herbarium at Andhra University. A total of 100 species have been recorded and enumerated with its family name, local name, part used, disease/ailment and uses (table 1).

Table. 1. Medicinal Plants and their uses.

\begin{tabular}{|c|c|c|c|c|}
\hline S.No & Plant name & Local name & Distribution & Traditional uses \\
\hline 1. & $\begin{array}{l}\text { Acacia chundra (Roxb.ex } \\
\text { Rotti.)Willd. (Mimosaceae) } \\
\text { Habit: A moderate sized tree }\end{array}$ & Pikkachandra & $\begin{array}{l}\text { Fairy common in } \\
\text { thorny scrub } \\
\text { jungles }\end{array}$ & Stem bark: Diabetes \\
\hline 2. & $\begin{array}{l}\text { Acacia leucophloea } \\
\text { (Roxb.)Willd. (Mimosaceae) } \\
\text { Habit:A Moderate sized tree }\end{array}$ & Tellatumma & $\begin{array}{l}\text { Common in dry } \\
\text { deciduous forests }\end{array}$ & $\begin{array}{l}\text { Stem bark: Dysentery, } \\
\text { wound }\end{array}$ \\
\hline 3. & $\begin{array}{l}\text { Acacia nilotica }(\text { Linn.)Willd.ex } \\
\text { Del.(Mimosaceae) } \\
\text { Habit: A Moderate sized tree }\end{array}$ & Nallathumma & $\begin{array}{l}\text { Common in } \\
\text { waste lands and } \\
\text { cultivated }\end{array}$ & $\begin{array}{l}\text { Stem bark: Bone fracture. } \\
\text { Menstrual complaints, } \\
\text { piles, snake bite }\end{array}$ \\
\hline 4. & $\begin{array}{l}\text { Acanthospermum hispidum } \\
\text { DC.(Asteraceae) } \\
\text { Habit: erect hispid herb }\end{array}$ & Palleru & $\begin{array}{l}\text { Common weed in } \\
\text { waste places }\end{array}$ & $\begin{array}{l}\text { Root: Leprosy, } \\
\text { leucorrhoea, menorrhagia }\end{array}$ \\
\hline 5. & $\begin{array}{l}\text { Adiantum incisum } \\
\text { Forssk.(Adiantaceae) } \\
\text { Habit: A leafy fern }\end{array}$ & Jerrikura & $\begin{array}{l}\text { Common along } \\
\text { the stream banks } \\
\text { and moist shady } \\
\text { areas }\end{array}$ & $\begin{array}{l}\text { Whole plant: Scorpion } \\
\text { sting }\end{array}$ \\
\hline 6. & $\begin{array}{l}\text { Ailanthus excelsa } \\
\text { Roxb.(Simaroubaceae) } \\
\text { Habit: large deciduous tree }\end{array}$ & Peddamanu & $\begin{array}{l}\text { A frequent } \\
\text { member of } \\
\text { deciduous forests }\end{array}$ & $\begin{array}{l}\text { Stem bark: Blood } \\
\text { pressure, diarrhea, } \\
\text { dysentery, fever, } \\
\text { leucorrhoea, piles }\end{array}$ \\
\hline 7. & $\begin{array}{l}\text { Aloe barbadensis Mill.(Liliaceae) } \\
\text { Habit:Perennial herb }\end{array}$ & Kalabanda & $\begin{array}{l}\text { Occasional along } \\
\text { the rocky hill } \\
\text { slopes and also } \\
\text { cultivated }\end{array}$ & $\begin{array}{l}\text { Root: Jaundice } \\
\text { Leaf: Pain, ulcer, wound } \\
\text { Mucilage: Boil, eye } \\
\text { diseases }\end{array}$ \\
\hline 8. & $\begin{array}{l}\text { Amorphophallus paeonifolius } \\
\text { (Dennst.) } \\
\text { (Araceae)Habit: Herb }\end{array}$ & Adavikanda & $\begin{array}{l}\text { Occasional in the } \\
\text { hills of Visakha } \\
\text { patnam district }\end{array}$ & $\begin{array}{l}\text { Stem: Leucorrhoea, } \\
\text { menorrhagia, piles }\end{array}$ \\
\hline 9. & $\begin{array}{l}\text { Aristolochia bracteolata Lamk. } \\
\text { (Aristolochiaceae) } \\
\text { Habit: A perennial prostrate herb }\end{array}$ & Gadidagadapa & $\begin{array}{l}\text { Common weed } \\
\text { of cultivated } \\
\text { fields, waste } \\
\text { places }\end{array}$ & $\begin{array}{l}\text { Root: Dental problems } \\
\text { Leaf: Skin diseases } \\
\text { Whole plant: } \\
\text { Anthelmintic, purgative }\end{array}$ \\
\hline 10. & $\begin{array}{l}\text { Azima tetracantha } \\
\text { Lam.(Salvadoraceae) } \\
\text { Habit: Thorny shrub }\end{array}$ & Tellavuppi & $\begin{array}{l}\text { Common in the } \\
\text { outskirts of } \\
\text { thorny scrub } \\
\text { jungles }\end{array}$ & Root: Stomache disorders \\
\hline 11. & $\begin{array}{l}\text { Baliospermum montanum } \\
\text { (Willd.)Muell. } \\
\text { (Euphorbiaceae) } \\
\text { Habit:A stout erect under shrub }\end{array}$ & Chittamudamu & $\begin{array}{l}\text { Occasional in the } \\
\text { undergrowth of } \\
\text { moist deciduous } \\
\text { forests }\end{array}$ & $\begin{array}{l}\text { Root: Pain, stomache } \\
\text { disorders }\end{array}$ \\
\hline 12. & $\begin{array}{l}\text { Bambusa arundinacea Willd. } \\
\text { (Poaceae) } \\
\text { Habit: A tall thorny culm }\end{array}$ & Veduru & $\begin{array}{l}\text { Fairy common } \\
\text { along hill slopes } \\
\text { of forests }\end{array}$ & $\begin{array}{l}\text { Stem: Bone fracture, } \\
\text { lymph adenitis, } \\
\text { rheumatism }\end{array}$ \\
\hline 13. & $\begin{array}{l}\text { Barringtonia acutangula (Linn.) } \\
\text { Gaertn. (Barringtoniaceae) }\end{array}$ & Tarrepu & $\begin{array}{l}\text { A common } \\
\text { species along }\end{array}$ & $\begin{array}{l}\text { Root: Rheumatism } \\
\text { Stem bark: Blood }\end{array}$ \\
\hline
\end{tabular}


Habit: An evergreen tree

14. Bauhinia vahlii W. \&

A.(Caesalpiniaceae)

Habit: A large woody climber

15. Buchanania lazan Sperng.

(Anacardiaceae)

Habit: A medium sized tree

16. Caesalpinia bonduc (Linn.)

Roxb.

(Caesalpiniaceae)

Habit: A large scandent prickly

shrub

17. Capparis zeylanica Linn.

(Capparaceae)

Habit: Throny climbing shrub

18. Cassia auriculta Linn.

(Caesalpinaceae)

Habit: A much branched shrub

19. Cassine glauca (Roth.) O.

Kuntze. (Celastraceae)

Habit: Small tree

20. Celastrus paniculatus Willd.

(Celastraceae)

Habit: Climbing shrub

21. Chloroxylon swietenia DC.

(Rutaceae)

Habit: An erect tree

22. Cipadessa baccifera (Roth.) Miq. (Meliaceae)

Habit: A Bushy shrub

23. Cissampelos pareira Linn. Var. hirsuta (Buch-Ham.ex

DC.)Forman.

(Menispermaceae)

Habit: A climbing under shrub

24. Cissus pallida (W. \& A.) Planch.

(Vitaceaae)

Habit: An erect shrub

25. Cleistanthus collinus (Roxb.) Vodisa

Benth. Ex. Hook. f.

(Euphorbiaceae)

Habit: Small deciduous tree

26. Clerodendrum serratum (Linn.)

Moon. (Verbenaceae)

Habit: Woody perennial shrub

27. Clerodendrum viscosum

Moldenke (Verbenaceae)

Habit: Gregarious under shrub

28. Coldenia procumbens Linn.

(Boraginaceae)

Habit: Prostrate scabrid herb

29. Colebrookea oppositifolia Sm.

(Lamiaceae)
Addaku

Charapappu

Gaccha

Adonda

Nelatangedu

Pantamanu

Teegapalleru

Billudu

Paradonda

Gundapaku

Budaritiga

Bommalamarri

Piduduru

Hamsa padi

Joldi streams and on swampy land

In deciduous forests

Common in deciduous forests

On the foot hills of scrub jungles

Common in

forests and

hedges

Common and
abundant at
forest

Common in forests

Throughout on hills.

Frequent in deciduous forest areas

Common near villages and dry forests

Common on hedges, on bushes along edges of the

forests

Common in dry forests

Common in dry open forests

Common in the hilly areas along ghat areas

Common in the undergrowth of deciduous forests

\section{Commonly} occuring along bunds of streams in dried ditches and tanks

Occasional along the hill slopes in pressure, bronchial asthma, diarrhea,

dysentery, piles

Root: Leprosy, stomach

disorders

Leaf: Dropsy

Stem bark: Diarrhoea,

dysentery, malaria

Stem bark: Bone fracture, dysentery, pain

Root: Dysentery, stomach disorders

Root bark: Epilepsy

Stem bark: Diarrhoea

Leaf: Hydrocele

Seed: Headache

Root: Aphrodisiac

Root bark: Pain

Root: Antiemetic, diarrhea

Leaf: Burn, eye diseases

Stem bark: Acidity,

dysentery, jaundice

Stem bark: Sterility, pain, scorpion sting

Root: Venereal diseases

Leaf: Headache

Seed oil: Hair care, skin

diseases

Root bark: Impotence

Leaf: Wound

Stem bark: Bone fracture,

dental problems

Root: Laxative

Stem bark: Rheumatism

Root: Antiemetic, diarrhea, migraine, stomache disorder

Leaf: Acidity

Root: Boil, warts

Stem bark: Menorrhagia

Root: Fever, leprosy, menstrual complaints, skin diseases

Leaf: Headache

Leafy: Ear complaints

Root: Paralysis

Leaf: Rheumatism

Whole plant: Cracks in

feet, cuts, skin diseases

Leaf: Bruise, wound 
Habit: Shrub

30. Combretum roxburghii Spreng.

(Combretaceae)

Habit: A large climbing shrub

31. Crataeva magna (Lour.) DC

(Capparaceae)

Habit: A medium sized deciduous tree

32. Cryptolepis buchananii Roem \& Schult. (Asclepiadaceae)

Habit: A large twinning shrub

33. Dalbergia lanceolaria Linn.f.

(Fabaceae)

Habit: A large deciduous tree

34. Dalbergia latifolia

Aroxb.(Fabaceae)

Habit: A Large deciduous tree

35. Dalbergia volubilis Roxb.

(Fabaceae)

Habit: A large woody climber

36. Decalepis hamiltonii Wt.\& Arn. (Asclepiadaceae)

Habit: Climbing shrub

37. Dendrophthoe falcata (Linn.)

Etting. (Loranthaceae)

Habit: Shrubby partial stem parasite

38. Desmodium gangeticum (Linn.)

DC. (Fabaceae)

Habit: an erect diffusely branched under shrub

39. Dillenia indica Linn.

(Dilleniaceae)

Habit: A medium to large sized tree

40. Dillenia pentagyna Roxb.

(Dilleniaceae)

Habit: A large deciduous tree

41. Diospyros melanoxylon Roxb.

(Ebenaceae)

Habit: A moderate sized tree

42. Ecbolium viride (Forssk.) Alston

(Acanthaceae)

Habit: Small shrub

43. Entada pursaetha DC.

(Mimosaceae)

Habit: A large woody climber

44. Erythroxylum monogynum Roxb. (Erythroxylaceae)

Habit: Small tree

45. Feronia limonia (Linn.) Swingle

(Rutaceae)

Habit: Tree

46. Ficus benghalensis Linn. Marri

(Moraceae)

Habit: Evergreen tree

47. Ficus heterophylla Linn. f. Kuvva juvvi

(Moraceae)

Habit: Scandent shrub

48. Ficus hispida Linn. f. (Moraceae)

Habit: A moderate sized tree
Suritithivva

Velimirichettu

Adavipalatiga

Irugudu

Virugudu

Thiyyatangedu

Barrisungandhi

Radam

Gitanaram

Revadi

Pedda Revadi

Tumikaku

Ekanga

Gillatiga

Devadaru

Velaga

Buddamedi the moist

deciduous forests

Common in

deciduous forests

on trees and

shrubs

Frequent along

river bunds

Frequent at foot

hills in shady

areas

Common in dry

deciduous forests

Common in

deciduous forests

Common in

deciduous forests

Rare in forests

A frequent

parasite on all

deciduous trees

Common in dry

forests

Frequent along

moist hilly areas

Frequent in forest areas

Common in dry deciduous forests

Common in

hedges and

bushes of dry

deciduous forests

Common climber

in the hilly areas

Common in the deciduous forests

In open dry forests

Common avenue tree along road

sides

Common near streams and

rivers

Frequent along

the streams in the hills
Leaf: Boil, skin diseases

Stem bark: Dysentery

Root: Stomach disorders

Root: Acidity

Whole plant: Rickets

Stem bark: Fever, paralysis, rheumatism, stomach disorders Stem bark: Stomach disorders Seed: Rheumatism Stem bark: Stomach disorders

Root: Apthous ulcers, tonic

Stem bark: Piles Whole plant: Bone fracture, diarrhoea, leucorrhoea

Root: Boil, bronchial asthma, cough, whoping cough

Calyx: Stomach disorders

Stem bark: Paralysis, snake bite

Leaf: Diarrhoea, tonic Stem bark: Bone fracture, cold, cough

Root: Dysuria

Fruit: jaundice

Stem bark: Antiemetic, diarrhoea

Seed: heapatic complaints Cotyledons: Anthelminitic Leaf: Anthelmentic, antiemetic, jaundice

Root: Rheumatism, whooping cough Stem bark: Pain Prop root: Diarrhoea Stem bark: Boil, menstrual complaints

Root: Bronchial asthma

Root bark: Cough

Leaf: Spermtorrhoea

Stem bark: Diarrhoea,

fever

Latex: Warts 


\section{Ficus religiosa Linn. (Moraceae) Ravi}

Habit: A large tree

50. Ficus semicordata Buch. Ham. ex Verubodda Sm (Moraceae)

Habit: Medium sized tree

51. Garuga pinnata Roxb.

(Burseraceae)

Habit: A large tree

52. Glycosmis mauaritiana (Lamk.) Golugu

Tanaka (Rutaceae)

Habit: Shrub

53. Gmelina arborea Roxb.

(Verbenaceae)

Habit: Straggling shrub

54. Gmelina asiatica Linn.

(Verbenaceae

Habit: A large deciduous tree

55. Gnetum ula Brongn. (Gnetaceae)

Habit: A large woody climber

56. Grewia rothii DC. (Tiliaceae)

Habit: An erect shrub

57. Gymnema sylvestre $\mathrm{R}$. Br.

(Asclepiadaceae)

Habit: Climbing shrub

58. Hemidesmus indicus (Linn.)

R.Br. (Asclepiadaceae)

Habit: Twinning shrub

59. Holarrhena pubescens (Buch. Ham.) Wall. (Apocynaceae)

Habit: A small deciduous tree

60. Holoptelea integrifolia (Roxb.)

Planch (Ulmaceae)

Habit: A large deciduous tree

61. Hugonia mystax Linn. (Linaceae)

Habit: A rambling shrub

62. Hybanthus enneaspermus

(Linn.f.) Muell. (Violaceae)

Habit: Small annual herb

63. Hymenodictyon orixense (Roxb.)

Mabb. (Rubiaceae)

Habit: A large deciduous trees

64. Ichnocarpus frutescens $\mathrm{R} . \mathrm{Br}$.

(Apocynacae)

Habit: A Climbing shrub

65. Jasminum angustifolium (Linn.)

Willd (Oleaceae)

Habit: Climbing shrub

66. Lannea coromandelica (Houtt.)

Merr. (Anacardiaceae)

Habit: Deciduous tree

67. Leea indica Merr. (Leeaceae)

Habit: A large shrub or small tree

68. Leptadenia reticulata (Retz.) Wt. \& Arn. (Asclepiadaceae)
Avenue or

roadside tree

Garugudu

Gummudu

Gummudu

Lalloditiga

Jibilika

Podapatri

Sugandhipala

Kodisapala

Nemalinaara

Geddagoru

Ratnapurusha

Dudipala

Palatiga

Adavimalli

Gumpena

Konda mookudu

Mukkutummudu
In moist

deciduous forests

Common on hill slopes

Frequent species on the outsksirts of forests

Common in the open forest areas

Fairly common on the hill slopes

Rare along stream banks in the moist deciduous forests Common in the forest hilly areas Frequent on the outskirts of forests

Occasional both in plains and forests

Common in the deciduous forests

Occasional on hill slopes

In dry deciduous forest areas

Frequently appears on sandy soils and moist places

In the hilly region

In the plains and lower hilly areas

Commonly found indry deciduous forests

Commonly in deciduous forests of hilly areas Rare

Common in outskirts of
Stem bark: Blood

pressure, diarrhea, dysentery, menstrual complaints, pain Stem bark: Fertility

Stem bark: Bone fracture, menstrual complaint, pain

Root: Cough

Stem bark: Bone fracture, bronchial asthma, cough, diarrhea, epilepsy, stomach disorders Fruit: Dandruff

Stem bark: Diarrhoea

Root bark: Dysentery, venereal diseases Leaf: Diabetes, snake bite

Root: Antiemetic, ulcers, cut, diarrhea, fever, fit, jaundice, skin diseases Leaf: Insect bite Root \& Stem bark: Dysentery, diarrhea

Stem bark: Ant fertility, bone fracture, dental problems, dysentery, fever, pain, piles, rheumatism, stomach disorders

Root: Dropsy, epilepsy, leucorrhoea, menorrhea, venereal diseases Root: Impotence, leucorrhoea, menorrhea, rheumatism

Whole plant: Stomach disorders

Stem bark: Leucorrhoea, menorrhoea

Root: Jaundice, snake bite

Root: Stomach disorders

Stem bark: Cut, dysentery, fever, pain, stomach disorders

Root: Diarrhoea, dysentery, headache Leaf and root: In skin affection, wounds 
Habit: A climbing shrub

69. Litsea glutinosa (Lour.) Robinson Naramamidi

(Lauraceae)

Habit: An evergreen shrub or tree

70. Litsea monopetala (Roxb.) Pers.

(Lauraceae)

Habit: Medium sized tree

71. Macaranga peltata (Roxb.)

Muell.-Arg. (Euphorbiaceae)

Habit: Medium sized tree

72. Madhuca longifolia (Koen.)

MacBride (Sapotaceae)

Habit: A large tree

73. Malluotus philippinensis (Lamk.) Muell.-Arg. (Euphorbiaceae)

Habit: Small tree

74. Melastoma malabathricum Linn. Nitidanimma

(Melastomataceae)

Habit: Shrub

75. Mirabilis jalap Linn.

(Nyctaginaceae)

Habit: A tall herb

76. Mitragyna parvifolia (Roxb.)

Korth. (Rubiaceae)

Habit: A large deciduous tree

77. Mucuna monosperma DC.ex

Wight (Fabaceae)

Habit: A large woody climber

78. Mucuna pruriens (Linn.) DC.

(Fabaceae)

Habit: A slender climber

79. Murraya koenigii (Linn.) Sperng. (Rutaceae)

Habit: Small aromatic tree

80. Naravelia zeylanica DC.

(Ranunculaceae)

Habit: Climbing shrub

81. Naringi crenulata (Roxb.)

Nicolson (Rutaceae)

Habit: Small tree

82. Oroxylum indicum (Linn.)

Benth.ex Kurz (Bignoniaceae)

Habit: medium sized tree

83. Pavetta tomentosa Roxb. ex Sm.

(Rubiaceae)

Habit: Shrub

84. Pergularia daemia (Forssk.)

Choiv. (Asclepiadaceae)

Habit: Perennial climber

85. Plumbago indica Linn.

(Plumbaginaceae)

Habit: Perennial shrub

86. Plumbago zeylanica Linn.

(Plumbaginaceae)

Habit: Scandant undershrub

87. Pterocarpus marsupium Roxb.

(Fabaceae)

Habit: A large deciduous tree

88. Rauvolfia serpentina (Linn.)
Chinamamidi

Palakachettu

Ippa

Pandrakachettu

Erramogamalle

Bandari

Pulugillatiga

Duradagondi

Karivepa

Vorratiga

Torrivelaga

Pampini

Kondapapidi

Dustapatiga

Errachitramulamu

Rare in

Visakhapatnam

Tellachitramulamu

Occasional in waste lands

Virugudu

Common in the

hills in moist regions

moist deciduous

forests

Common along

the moist valleys

Common alon

hill slopes and

outskirts of

forests

Comen

ub jungles

Planted for its

showy flowers

Occational in

Rarely occurred

in the interior

Frequently seen

climbing on

shrubs and

Cues

run wild in the

forests

Frequent near in the

tskirts of

Common dry

forests

Mostly in the outskirts of

forests

hilly regions

Common in

waste places
Root: wounds

Stem bark: Boil fracture,

leucorrhoea

Stem bark: Rheumatism

ccasional along

deciduous forests

Occasional in
Fruit: Bone fracture

Root: Epilepsy, leprosy

Leaf: Burn

Stem bark: Cough,

diarrhoea

Root: Epilepsy

Stem bark: Diarrhoea

Stem bark: Leucorrhoea

Root: Blood pressure, leucorrhoea, menorrhoea, piles, stomach disorders Stem bark: Dysentery, stomach disorders

Root: Dysmenorrhoea

Seeds: tonic

Roots: epilepsy

Leaf: dental problems

Root: Dysentery

Leaf: In dysentery, fever

Leaf: Cold

Stem bark: Bone fracture, dysentery, fever

Root: Stomach disorders Stem bark: Dysentery, ear complaints

Root: Alexeteric, fever

Root: Cold, cough, dysentery, fever, skin diseases

Leaf: Diarrhea, eye

diseases

Root: Abortifacient,

stomach disorders

Root bark: Fever, pain

Root: Abortifacient, bone

fracture, pain, rheumatism

Leaf: Boil, skin disease, sore

Stem bark: Cough, dental problems, dysentery

Root: Diabetes, diarrhea, 


\begin{tabular}{|c|c|c|c|c|}
\hline & $\begin{array}{l}\text { Benth. ex Kurtz (Apocynaceae) } \\
\text { Habit: Small undershrub }\end{array}$ & & $\begin{array}{l}\text { moist deciduous } \\
\text { forest }\end{array}$ & $\begin{array}{l}\text { fever, scorpion sting, skin } \\
\text { disease }\end{array}$ \\
\hline 89. & $\begin{array}{l}\text { Schleichera oleosa (Lour.)Oken. } \\
\text { (Sapindaceae) } \\
\text { Habit: A large tree }\end{array}$ & Bushi & $\begin{array}{l}\text { Common in } \\
\text { moist hill slopes }\end{array}$ & $\begin{array}{l}\text { Stem bark: Bone fracture, } \\
\text { dysentery, malaria }\end{array}$ \\
\hline 90. & $\begin{array}{l}\text { Semecarpus anacardium Linn.f. } \\
\text { (Anacardiaceae) } \\
\text { Habit:A moderate sized tree }\end{array}$ & Nallajidi & $\begin{array}{l}\text { Common in dry } \\
\text { deciduous forests }\end{array}$ & $\begin{array}{l}\text { Stem bark: Cut, diarrhea, } \\
\text { fever, leucorrhoea, } \\
\text { malaria, rheumatism, piles }\end{array}$ \\
\hline 91. & $\begin{array}{l}\text { Terminalia alata Heyne ex. Roth. } \\
\text { (Combretaceae) } \\
\text { Habit: A large tree }\end{array}$ & Nallamaddi & $\begin{array}{l}\text { Common } \\
\text { member of } \\
\text { deciduous forests }\end{array}$ & $\begin{array}{l}\text { Stem bark: Cardiac } \\
\text { complaint, piles }\end{array}$ \\
\hline 92. & $\begin{array}{l}\text { Urginea indica (Roxb.)Kunth } \\
\text { (Liliaceae) } \\
\text { Habit: Bulbous herb }\end{array}$ & Kondavulli & $\begin{array}{l}\text { Occasional in } \\
\text { open dry hilly } \\
\text { slopes }\end{array}$ & Bulb: Boil, paralysis \\
\hline 93. & $\begin{array}{l}\text { Vanda tessellata (Roxb.)HK ex. } \\
\text { G.Don. (Orchidaceae) } \\
\text { Habit: Epiphytic herb }\end{array}$ & Radam & $\begin{array}{l}\text { Common on the } \\
\text { trees in the hilly } \\
\text { forest areas }\end{array}$ & $\begin{array}{l}\text { Leaf: ear complaints } \\
\text { Stem: bone fracture }\end{array}$ \\
\hline 94. & $\begin{array}{l}\text { Vernonia cineria (Linn.)Less. } \\
\text { (Asteraceae) } \\
\text { Habit: Herb }\end{array}$ & Sahadevi & $\begin{array}{l}\text { Common weed } \\
\text { along road sides }\end{array}$ & $\begin{array}{l}\text { Root: Snake bite } \\
\text { Root bark: Menstrual } \\
\text { complaints }\end{array}$ \\
\hline 95. & $\begin{array}{l}\text { Wattakaka volubilis (Linn.)Stapf. } \\
\text { (Asclepiadaceae) } \\
\text { Habit: A large climbing shrub }\end{array}$ & Bandigurijaaku & $\begin{array}{l}\text { Common in } \\
\text { waste places }\end{array}$ & $\begin{array}{l}\text { Leaf: Scorpion sting, tonic } \\
\text { Root \& Leaf: Snake bite }\end{array}$ \\
\hline 96. & $\begin{array}{l}\text { Woodfordia fruticosa (Linn.) } \\
\text { Kurz. (Lythraceae) } \\
\text { Habit: A bushy shrub }\end{array}$ & Adavijaji & $\begin{array}{l}\text { Common in } \\
\text { deciduous forests }\end{array}$ & $\begin{array}{l}\text { Flowers: Dysentery, } \\
\text { leucorrhoea, skin disease, } \\
\text { piles, diarrhea, ulcer }\end{array}$ \\
\hline 97. & $\begin{array}{l}\text { Ximenia americana Linn. } \\
\text { (Olacaceae) } \\
\text { Habit: Large spinous herb }\end{array}$ & Nakkera & $\begin{array}{l}\text { Common in dry } \\
\text { forests on stony } \\
\text { ground }\end{array}$ & $\begin{array}{l}\text { Root bark: Diarrhoea } \\
\text { Fruit: stomach disorders }\end{array}$ \\
\hline 98. & $\begin{array}{l}\text { Xantolis tomentosa (Roxb.)Raf. } \\
\text { (Sapotaceae) } \\
\text { Habit: Moderate sized tree }\end{array}$ & Paala gotti & $\begin{array}{l}\text { In forests of } \\
\text { kokkira palli }\end{array}$ & Fruit: Skin bite \\
\hline 99. & $\begin{array}{l}\text { Ziziphus mauritiana Lam. } \\
\text { (Rhamnaceae) } \\
\text { Habit: Much branched thorny tree }\end{array}$ & Gangaregu & $\begin{array}{l}\text { Found in all } \\
\text { scrub and dry } \\
\text { deciduous forests }\end{array}$ & Root: Cold, fever \\
\hline 100. & $\begin{array}{l}\text { Ziziphus xylopyrus (Retz.) Willd } \\
\text { (Rhamnaceae) } \\
\text { Habit: Small tree or large } \\
\text { straggling shrub }\end{array}$ & Gottika & $\begin{array}{l}\text { Frequent in open } \\
\text { hilly areas }\end{array}$ & $\begin{array}{l}\text { Root: Fever, malaria } \\
\text { Stem bark: Antiemetic, } \\
\text { cholera, dysentery, } \\
\text { stomach disorders }\end{array}$ \\
\hline
\end{tabular}

\section{Result \& Discussion}

The present investigation comprises 100 species of phyto-medicinal plant species belonging to 85 genera and 53 families of Visakhapatnam Tribal area. Out of 100 species Angiosperm are 51, Gymnosperms 1, and Pteridophytes 1. Out of 51 angiosperms 47 are Dicots and Monocots are 4 families. For each species botanical name, family, local name, parts used, distribution of the species and ailments treated are provided. Traditional healers are using these plants to cure many diseases like stomachache, diarrhea, headache, fertility, problems skin problems, cold, fever, cough, jaundice, wounds, diabetes, asthma, bone fractures, piles, snake and scorpion bites etc., Trees are 43 , shrubs are 37, herbs are 12 and climbers are 8 species found to be the study area. The most dominant families in the study were, Fabaceae and Asclepiadaceae 7, Rutaceae and Moraceae 5, Mimosaceae and Verbenaceae 4, Euphorbiaceae, Apocinaceae, Anacardiaceae, Caesalpiniaceae and Rubiaceae each one 3, remaining families have each one single species. Depending upon the plant parts used root and root bark is used in the 54, followed by stem and stem bark 47, leaf 25, and whole plant/flower/seed/latex are 17. Most of the herbal remedies are taken orally.

\section{Conclusion}

It can be concluded that the local and tribal people of the district have very good knowledge on the use of medicinal plants. But such knowledge of medicinal plants is restricted to a few persons in a rural area. The destructive harvesting of the medicinal plants by the maximum use of underground parts from the wild may lead to extinction of the species in the future. As demand for medicinal plants are ever increasing and these resources depleting from the nature. Therefore, there is a need to generate awareness among the local communities towards the sustainable utilization and conservation of medicinal plants. 


\section{Acknowledgment}

Authors are thankful to the authorities of Andhra Pradesh forestry for permission and help during explorations.

\section{Reference}

[1]. Reddy, C.S., K.N. Reddy \& S.N. Jadhav, Medicinal uses of Hildegardia populifolia and Pterocarpus santalinus: Red listed and endemic taxa in Andhra Pradesh. EPTRI-ENVIS News letter, 2000. 6(1): 9-10.

[2]. Jadhav, S.N., D.K. Ved., Utkesh Ghate, K.N. Reddy \& Ch.S. Reddy, Proceedings of the Workshop on Conservation Assessment and Management Planning for medicinal plants of Andhra Pradesh. Medicinal Plants Conservation Centre (MPCC), EPTRI, 2001, Hyderabad.

[3]. Reddy, C.S., K.N. Reddy \& S.N. Jadhav, Threatened (Medicinal) Plants of Andhra Pradesh. Medicinal Plants Conservation Centre, EPTRI, 2001. Hyderabad.

[4]. Jeevan, R \& R.R.V. Raju., Certain potential crude drugs used by tribals of Nallamalais, Andhra Pradesh for Skin diseases. Ethnobotany, 2001, 13: 110-115.

[5]. Reddy, C.S., K. Nagesh, K.N. Reddy \& V.S. Raju, Plants used in ethno veterinary practices by Gonds of Karimnagar district, Andhra Pradesh, India. J. Econ. Tax. Bot. 26: Chennai. Dec. $28^{\text {th }}-29^{\text {th }}, 2002$, Page: 19

[6]. Reddy, C.S. \& V.S. Raju, Folklore biomedicine for common veterinary diseases in Nalgonda district, Andhra Pradesh. Ethnobotany, 2000, 12: 113-117.

[7]. Jadhav, S.N. \& K.N Reddy, Threatened Medicinal Plants of Andhra Pradesh. ENVIS-SDNP Newsletter special issue, 2006, Pp 1828.

[8]. Gupta, V. G., S.J. Hussain \& S. Imam 1997. Medico-ethno botanical survey of Paderu forests of Araku valley, Andhra Pradesh, India. Fitoterapia, 1997, 68: 45-48.

[9]. Nisteswar, K. \& K. A. Kumar, Utilization values of medical-lore of Rampa Agency (Andhra Pradesh) in primary health care, Sachitra Ayurved. 1980, 33: 210-212

[10]. Rao, K.P. \& S. Harasreeramulu, Ethnobotany of selected medicinal plants of Srikakulam district, Andhra Pradesh. Anc. Sci. Life. 1985, 4:

[11]. Sudhakar, S. \& R.S. Rao, Medicinal plants of East Godavari district, Andhra Pradesh. J. Econ. Tax.Bot, 1985, 7: 399-406. $238-244$.

[12]. Aruneekumar, K., G. Satyanarayana, and K. Nisteswar, Medicinal plants of Kakinada, East Godavari District, and Andhra Pradesh. Indian Medicine, 1990, 2: 2-4.

[13]. Banerjee, D.K., Observations on the ethnobotany of Araku valley, Visakhapatnam district, Andhra Pradesh. J. Sci. Club, 1977, 31: $14-21$ 